\title{
Sensitivity of the orbital content of a model stellar system to the potential approximation used to describe it
}

\author{
D. D. Carpintero - F. C. Wachlin
}

Received: 7 December 2005 / Revised: 2 May 2006/

Accepted: 6 June 2006 / Published online: 10 October 2006

(C) Springer Science+Business Media B.V. 2006

\begin{abstract}
We have classified orbits in a stationary triaxial stellar system created from a cold dissipationless collapse of 100,000 particles. In order to integrate the orbits, two potential approximations with different fitting functions were used in turn. We found that the relative amount of chaotic versus regular orbits does depend on the chosen approximation of potential, even though both models resulted in very good fits of the underlying exact potential. On the other hand, the content of regular orbits, i.e., its distribution among main families, does not strongly depend of the potential approximation, being therefore a more robust signature of the gravitational system under study.
\end{abstract}

Keywords Triaxial stellar systems $\cdot$ Stellar orbits $\cdot$ Numerical methods

\section{Introduction}

In order to study the structure and evolution of stellar systems one can perform $N$-body simulations integrating the coupled equations of motion of the constituent particles, or one can try to construct analytic or numerical equilibrium models from the Poisson and the collisionless Boltzmann equations.

Self-consistent density distributions obtained from the particle approach can be of great help in determining the variety of orbits allowed by a certain system that results from solving the $N$-body problem. In particular, when dealing with collisionless systems like galaxies, it is possible to approximate the original (grainy) potential by a smooth (analytic) representation of it (see, e.g. Hernquist and Ostriker 1992), thus making it much faster to study the orbital structure of the smoothed system. This strategy relies on the presumable correspondence between characteristics (i.e., trajectories

\footnotetext{
D. D. Carpintero $(\varangle)$. F. C. Wachlin

Facultad de Ciencas Astronómicas y Geofísicas de la Universidad Nacional de La

Plata, and Consejo Nacional de Investigaciones Científicas y Tecnlógicas

Observatorio Astronómico, Paseo del Bosque S/N-1900 La Plata, Argentina

e-mail: ddc@fcaglp.unlp.edu.ar
} 
in the analytical potential) and the orbits of individual stars, although this point has never been proved rigorously (Kandrup and Sideris 2003). Anyway, the individual orbits allowed by a collisionless system constitute the basic building blocks for the dynamics of that system and by studying the orbital content of a potential we obtain very important clues about its structure and its relation to the equilibrium state.

The smoothing of $N$-body potentials has long been applied to stellar dynamics (see, e.g. Clutton-Brock 1973) allowing to represent a grainy density distribution by means of a set of functions that fits the overall aspect of that distribution. In this paper, we are particularly interested in generating a self-consistent $N$-body system which resembles an elliptical galaxy and that is in equilibrium in order to fit it by means of some functional representation. Once the fitting potential is obtained, orbits can be individually integrated and classified (Muzzio et al. 2005).

One may reasonably conjecture that the functional form of the fitting potential plays no major role in obtaining the orbital content, as long as the fitting is good. We will show that this is not the case: the fraction of chaotic orbits found in a given potential strongly depends on the details of the model. We will also show that, among regular orbits, the main families (boxes, long- and short-axis tubes) remain almost unaffected by minor differences in the potential.

\section{The model}

The $N$-body model upon which we worked was created by following the procedure described by Muzzio et al. (2005). First, a set of 100,000 particles were randomly distributed following a $1 / r$ density law inside a sphere of unit radius. Then the particle velocities were randomly generated from a Gaussian distribution, the dispersion of which was chosen so as to get an initial value of the random kinetic energy that was $0.25 \%$ of the initial value of the potential energy. Thus, according to Aguilar and Merrit (1990), such an initial setting would give rise to a highly triaxial system after the collapse has taken place.

All the particles were of the same mass, summing up a total mass equal to 1 . The gravitational constant $G$ was also chosen to be equal to 1 . After eliminating the unbound particles, the system was evolved for 10 crossing times ( $T_{\mathrm{cr}}$, equivalent to 0.574 time units) in order to let it relax. Rejecting some additional particles that represented some unrealistic extensions along the semimajor axis direction, the system was evolved again for another $10 T_{\mathrm{cr}}$.

By following this procedure, we ended up with a self-consistent triaxial ellipsoid of 86,818 particles that resembles an elliptical galaxy. In order to characterize its form, we introduce the usual notation of $a, b$ and $c$ for the major, middle and minor semiaxes of the distribution, respectively. For the system thus created, we obtained values of $b / a=0.75$ and $c / a=0.52$ when taking into account all particles, and $b / a=0.45$ and $c / a=0.35$ when only the $20 \%$ most strongly bound particles were considered (see Muzzio et al. 2005).

\section{Smooth representations of the $N$-body potential}

In order to integrate orbits in a collisionless version of this system, we replaced the $N$-body distribution of particles by a smooth representation of the potential generated 
by the mass distribution. To this end we introduced a quadrupolar approximation (Schwarzschild 1979; Muzzio et al. 2005) to write the smoothed potential:

$$
\Phi(r)=f_{00}(p)+f_{x x}(p) x^{2}-\left[f_{x x}(p)+f_{z z}(p)\right] y^{2}+f_{z z}(p) z^{2},
$$

where

$$
p^{2}=r^{2}+\varepsilon^{2}
$$

is the (squared) softened radius with softening parameter $\varepsilon$, which we set equal to 0.01 . We fitted the functions $f_{00}(p), f_{x x}(p)$ and $f_{z z}(p)$ using two different approaches. On the one hand we adopted expressions of the form (Muzzio et al. 2005):

$$
f(p)=\frac{C}{\left(p^{\kappa}+q^{\kappa}\right)^{\lambda}},
$$

with $C, \kappa, q$ and $\lambda$ constants to be obtained from the fitting. This model will be referred to as Model 1. On the other hand, we used different expressions:

$$
f(p)=C \exp \left\{\sum_{n=1}^{4} a_{n}[\ln (p+q)]^{n}\right\},
$$

with $C, a_{n}$ and $q$ constants, also to be obtained from the fitting. This potential model will be called Model 2.

Once the constants of the fitting functions were determined, we computed the value of the $N$-body potential at each point where there was a particle and compared it to the value obtained by using the smoothed potential representation. We found that the square root of the mean square percentual difference was $0.51 \%$ in the first case and $0.46 \%$ in the second case, so that we conclude that both approximations work very well, and at the same level of accuracy. On the other hand, the square root of the mean square percentual differences between the two different interpolating functions (evaluated at the positions of the particles of the $N$-body system) is $0.24 \%$ and Fig. 1 shows those fractional differences as a function of the potential of Model 1 divided by its value at the center of the system, so that the outermost particles lay at left and those closest to the center at right. We notice that the individual differences are of the same order of the dispersion throughout, except at the innermost and outermost regions where the differences reach about 1\%. (We thank Dr. J. C. Muzzio for providing us with the data concerning Fig. 1.)

In order to check whether the good fitting of the Model 2 is preserved when the system evolves, we repeated the procedure applied to Model 1 in Muzzio et al. (2005): the particles were allowed to move in the smoothed potential, and the fitting functions were evaluated from the distribution of particles at several times; the results from the initial instant and two other times were then combined together, the interpolating equations were fitted to them, and the square roots of the mean square percentual errors of the fittings, which constitute a measure of the structural changes suffered by the system in the time intervals considered, were then computed. Table 2 of Muzzio et al. (2005) shows that there were no significant differences among the dispersions obtained with Model 1; we found that the differences obtained with Model 2 are even smaller. Therefore, the system remains indeed stationary with either model.

There is still another question we must address. Even in a system in equilibrium, as the particles that make it up move, random changes in the density and, thus, in the potential will arise; these changes will be very small in a galaxy made up of, say, 


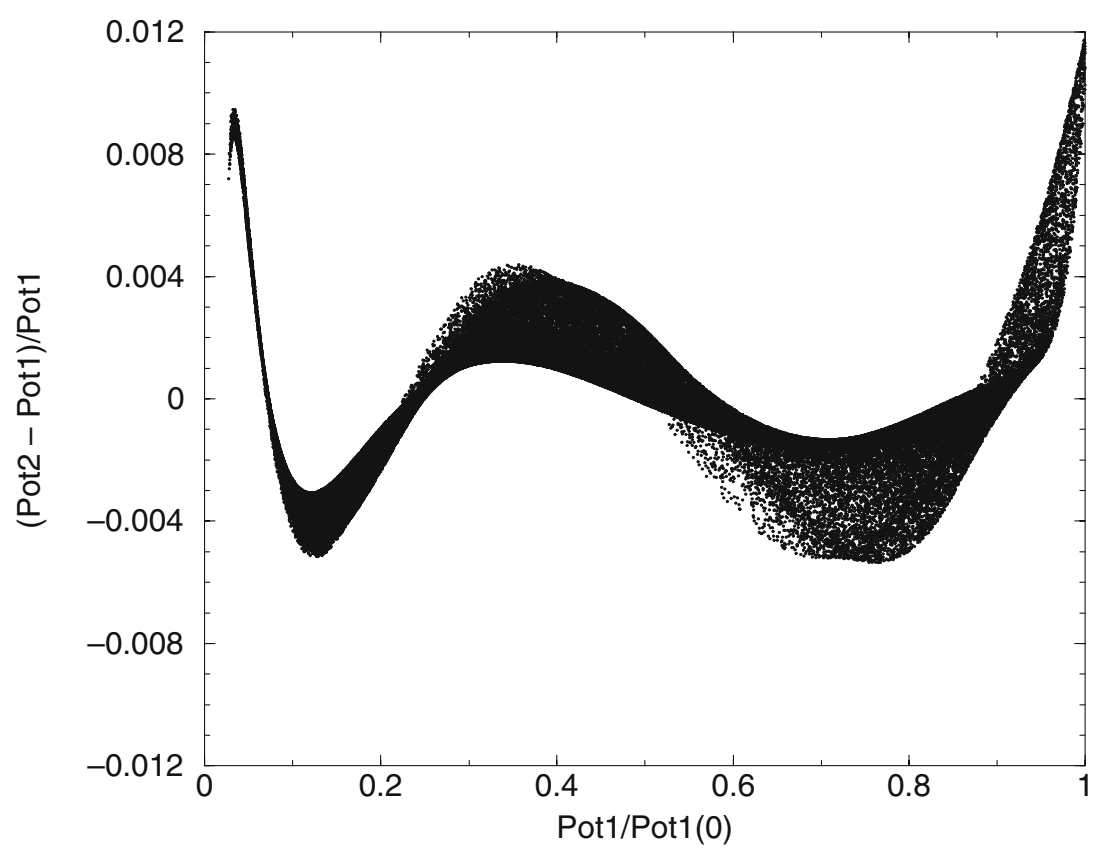

Fig. 1 The difference between the potentials of Models 1 and 2, relative to the former as a function of the potential of Model 1 normalized to its central value

$10^{11}$ stars, but more important in our system of about $10^{5}$ particles. This problem was investigated by Voglis et al. (2002), who found that less than 3\% of the orbits in their system changed their regular or chaotic character when the potential was fitted to $N$-body distributions corresponding to different times. With respect to our own system, Muzzio (submitted) compared the resulting regular/chaotic classification from the $N$-body distributions obtained combining the results at the initial time, $10 T_{\mathrm{cr}}$ and $20 T_{\mathrm{cr}}$ and at the initial time, $200 T_{\mathrm{cr}}$ and $400 T_{\mathrm{cr}}$; he found that there are no significant differences when the same mathematical expression is fitted to potentials determined at different times.

\section{Orbital classification}

In order to determine whether an orbit is regular or chaotic, we integrated it, computing at the same time its Lyapunov characteristic numbers. As in Muzzio et al. (2005), we regarded an orbit as regular whenever it had its two larger Lyapunov characteristic numbers smaller than a limiting value of 0.00155 (time units) ${ }^{-1}$, as partially chaotic when only its larger Lyapunov characteristic number is smaller than that value, and as totally chaotic otherwise.

In order to classify regular orbits in their families, we first used the frequency analysis automatic code of Carpintero and Aguilar (1998), but the frequencies were extracted with the aid of the algorithm implemented by Wachlin and Ferraz-Mello (1998). As in Muzzio et al. (2005), this improvement allows to compute the natural frequencies of the orbits with greater accuracy, at a cost of spectral resolution, 
i.e., two frequencies have to be more separated than a certain threshold $\Delta f$ in order to be properly identified. Thus, to be on the safe side, we merely discarded as not classified any orbit which happen to have a pair of frequencies closer than $\Delta f$. This, however, led to an undesirable consequence: too many (15.3\% in Model 1 and $15.9 \%$ in Model 2) of the orbits could not be classified. In order to reduce those percentages we reasoned that, on the one hand, one is mainly interested in low-resonant boxes (i.e., orbits the resonances of which are made up of small integers) and that, on the other hand, any pair of close frequencies, if in resonance, would yield a high-rank resonance (i.e., a resonance with large integers). Therefore, usually it makes no difference whether two close frequencies are extracted with high precision or not, because the orbit will end up being either a (non resonant) box or a high-resonant box, in which case it will be considered in practice as non resonant. We therefore decided to fed the unclassified orbits into a version of the code in which the discarding of orbits was not allowed, resulting that, effectively, all but a handful of orbits $(5 \%$ of the not classified, that is, less than $1 \%$ of the total) were classified as (non resonant) boxes. The price to pay, however, is that now we cannot safely assess how many resonances and independent frequencies are there in each orbit; therefore, again to be in the safe side, we classified regular orbits only into their main families: boxes and boxlets (BBL), long-axis tubes (LAT) and short-axis tubes (SAT).

\section{Results}

In Muzzio et al. (2005), a set of 3,472 initial conditions were chosen to be integrated in Model 1, in order to study the orbits generated by them. Here, we took the same set of 3,472 initial conditions and computed their orbits in Model 2. These orbits were then classified into regular, partially chaotic and totally chaotic as described above. The results are summarized in Table 1 .

The percentage of regular orbits is thus $47.35 \pm 0.85 \%$ in Model 1 and $40.96 \pm 0.83 \%$ in Model 2, a highly significant difference at the 5.4 $\sigma$ level. Alternatively, considering the chaotic orbits only, the percentages of fully chaotic orbits are, respectively, $83.15 \pm 0.88 \%$ and $85.37 \pm 0.78 \%$ for Models 1 and 2 , their difference amounts to just $1.9 \sigma$ and, thus, is probably not significant. (Here, and in the rest of the paper, the errors of the percentages are the dispersions derived from the binomial distribution, i.e., for a percentage $p$, obtained from a total number of data $N$, the dispersion is $\sigma=\sqrt{p(100-p) / N}$. $)$

We conclude that the sets of regular and chaotic orbits have effectively changed in shifting from one potential model to the other, even though the phase space initial conditions for the orbits were the same. This change can also be assessed by means of

Table 1 Number of regular, partially chaotic and totally chaotic orbits found in Model 1 (rows) and in Model 2 (columns)

\begin{tabular}{lllll}
\hline Model 1/Model 2 & Regular & Partially Chaotic & Fully Chaotic & Total \\
Regular & 1307 & 138 & 199 & 1644 \\
Partially chaotic & 70 & 118 & 120 & 308 \\
Fully chaotic & 45 & 44 & 1431 & 1520 \\
Total & 1422 & 300 & 1750 & 3472 \\
\hline
\end{tabular}


a crosstabulation analysis (Press et al. 1992), using Table 1 as a contingency table. In order to characterize the strength of association we used three different indicators: the Uncertainty Coefficient (Theil's) $U$, the Sakoda's Adjusted Contingency Coefficient (Pearson's) $C^{*}$, and the Association Coefficient (Cramer's) $V . U$ is the percent reduction in uncertainty in predicting the dependent (nominal) variable based on knowing the independent (nominal) variable (in our case, either rows or columns can be used as dependent or independent variables); $C^{*}$ and $V$, on the other hand, may be both interpreted as percentages of the maximum variation between the variables, although $V$ tends to be smaller than $C^{*}$ when computed from the same sample. The values obtained for Table 1 were $U_{1}=0.44, U_{2}=0.44, V=0.61$ and $C^{*}=0.80$, where $U_{1}$ and $U_{2}$ are the values considering in turn each potential model as the generator of the independent variable of the table. As can be seen, whatever indicator we want to choose, the level of association is relatively low, i.e., there is a non negligible change in going from one potential model to the other. It may still happen that the low strength of the observed association is not genuine, i.e., that the (low) association found is not different from what would be expected due to the chance of random sampling. To test the significance of the association, a $\chi^{2}$ test suffices; in our case, the probability of obtaining by chance our value of $\chi^{2}=2592.6$ with 4 degrees of freedom is less than $10^{-5}$, so the low association found is significant.

Let us now concentrate on the regular orbits, defined as the set of 1,307 orbits found as regular in both models. Table 2 was obtained classifying these orbits into the three main regular families, plus an additional category (Other) which includes not classified orbits as well as orbits classified as irregular by the automatic code. The percentages of boxes and boxlets, long-axis tubes, short-axis tubes and other orbits are, respectively, $77.96 \pm 1.15 \%, 2.37 \pm 0.42 \%, 19.20 \pm 1.09 \%$ and $0.46 \pm 0.18 \%$ in Model 1, and $78.12 \pm 1.14 \%, 2.37 \pm 0.42 \%, 19.13 \pm 1.09 \%$ and $0.38 \pm 0.17 \%$ in Model 2. The differences between the corresponding percentages of the two models are all well below the $1 \sigma$ level and show that, for regular orbits, both models give essentially the same results. As before, we can confirm these results by means of a crosstabulation analysis. We obtained strengths of association $U_{1}=0.95, U_{2}=0.95, V=0.86$ and $C^{*}=0.96$, with a probability of obtaining by chance the computed value of $\chi^{2}=2910.3$ with 9 degrees of freedom being less than $10^{-5}$. Thus, the two nominal variables are strongly associated, meaning that the main regular families are robust against small changes introduced in the potential representation.

As an illustration of the few cases in which a regular orbit shifted family, Fig. 2 shows the orbit 2140, one of the two orbits classified as a box orbit when integrated with Model 1, and as a SAT when the second model was used; $x, y$ and $z$ correspond, respectively, to the major, intermediate and minor axes of the potential. Clearly, the tube is very close to be a box, and a minimal change of the potential was enough to shift the original box orbit into the tube orbit.

Table 2 Number of orbits into each main regular family found in Model 1 (rows) and in Model 2 (columns)

\begin{tabular}{lllll}
\hline Model 1 / Model 2 & BBL & LAT & SAT & Other \\
BBL & 1017 & 1 & 0 & 1 \\
LAT & 0 & 30 & 0 & 1 \\
SAT & 2 & 0 & 249 & 0 \\
Other & 2 & 0 & 1 & 3 \\
\hline
\end{tabular}




\section{Discussion}

We may conclude that, as long as the mathematical expression of the interpolating function is not altered, the results obtained fitting it to the potential of the $N$-body system in equilibrium at different times are essentially the same. Alternatively, adjusting the potential with different mathematical expressions yields significantly different fractions of regular and chaotic orbits, even when the different expressions give equally good fittings. This result may be explained by the fact that, as it is well known, the chaotic behavior is very sensitive to changes in the potential. An extreme example is that of a fully regular system which becomes chaotic when a small perturbation is added; a less extreme one is the dependence of the amount of chaos on the energy in the Hénon-Heiles potential. However, as we have seen, the global structure and stability of the system are not affected by this effect. This is probably due to the non uniqueness of the equilibrium states of a stellar system, i.e., several distributions of stars in the phase space may yield systems with the same global features and the same global stability.
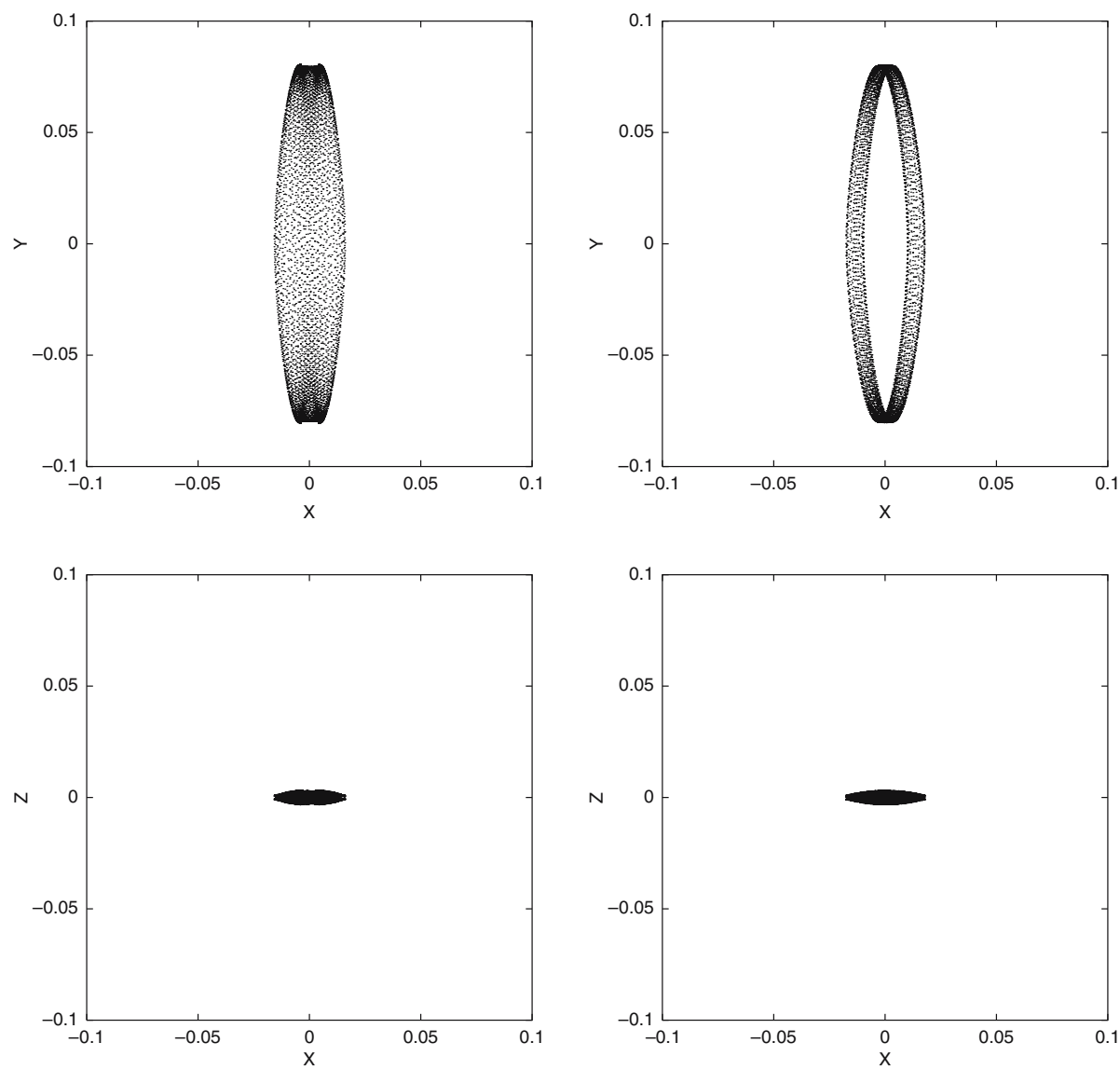

Fig. 2 Left: orbit integrated in the potential Model 1. Right: same initial condition integrated in the potential Model 2 
On the other hand, the distribution of regular orbits into families is robust: a small change of the potential neither destroys nor mixes families, but maintains the general regular structure. This result is valid as long as the orbits are well inside the regular regions; borderline zones, as we have just seen, are prone to switch between regular and chaotic behaviors. Recalling the KAM theorem, which states that a small, smooth, conservative perturbation applied to an integrable system produces only slight deformations to most invariant tori, one can conjecture that small perturbations applied to non integrable potentials will also have the same effect on the deep regular regions: a simple deformation of the invariant tori, which is precisely what we have found in our work.

Acknowledgements The comments of an anonymous referee were very useful to improve the original version of the present paper and are greatfully acknowledged. The authors wish to thank Dr. J. C. Muzzio for providing the data leading to Fig. 1. This work was supported with grants from the Universidad Nacional de La Plata, the Consejo Nacional de Investigaciones Científicas y Técnicas and the Agencia Nacional de Promoción Científica y Tecnológica de la República Argentina.

\section{References}

Aguilar, L.A., Merritt, D.: The structure and dynamics of galaxies formed by cold dissipationless collapse. Astrophys. J. 354, 33-51 (1990)

Carpintero, D.D., Aguilar, LA.: Orbit classification in arbitrary 2D and 3D potentials. Monthly Notices R. Astronomic. Soc. 298, 1-21 (1998)

Clutton-Brock, M.: The gravitational field of three dimensional galaxies. Astrop. \& Sp. Science 23, 55-69 (1973)

Hernquist, L., Ostriker, J.P.: A self-consistent field method for galactic dynamics. Astrophys. J. 386, 375-397 (1992)

Kandrup, H.E., Sideris, I.V.: Smooth potential chaos and $N$-body simulations. Astrophys. J. 585, 244-249 (2003)

Muzzio, J.C., Carpintero, D.D., Wachlin, F.C.: Spatial structure of regular and chaotic orbits in a self-consistent triaxial stellar system. Celest. Mech. Dynam. Astron. 91, 173-190 (2005)

Press, W.H., Teukolsky, S.A.,Vetterling, W.T., Flannery, B.P.: Numerical recipes: the art of scientific computing. Cambridge University Press, Cambridge (1992)

Schwarzschild, M.: A numerical model for a triaxial stellar system in dynamical equilibrium. Astrophys. J. 232, 236-247 (1979)

Voglis, N., Kalapotharakos, C., Stavropoulos, I.: Mass components in ordered and in chaotic motion in galactic $N$-body models. Monthly Notices R. Astronomic. Soc. 337, 619-630 (2002)

Wachlin, F.C., Ferraz-Mello, S.: Frequency map analysis of the orbital structure in elliptical galaxies. Monthly Notices R. Astronomic. Soc. 298, 22-32 (1998) 